\title{
ON FINDING FIELDS FROM THEIR ALGEBRAIC CLOSURE GEOMETRIES
}

\author{
KITTY L. HOLLAND
}

(Communicated by Andreas R. Blass)

\begin{abstract}
It is shown that if $F_{1}$ and $F_{2}$ are algebraically closed fields of nonzero characteristic $p$ and $F_{1}$ is not isomorphic to a subfield of $F_{2}$, then $F_{1}$ does not embed in the skew field of quotients $O_{F_{2}}$ of the ring of morphisms of the additive group of $F_{2}$. From this fact and results of Evans and Hrushovski, it is deduced that the algebraic closure geometries $G\left(K_{1} / F_{1}\right)$ and $G\left(K_{2} / F_{2}\right)$ are isomorphic if and only if $K_{1}: F_{1} \simeq K_{2}: F_{2}$. It is further proved that if $F_{0}$ is the prime algebraically closed field of characteristic $p$ and $F$ has positive transcendence degree over $F_{0}$, then $O_{F}$ and $O_{F_{0}}$ are not elementarily equivalent.
\end{abstract}

\section{INTRODUCTION}

If $F$ and $K$ are algebraically closed fields with $F$ a subfield of $K$, the set of algebraically closed extensions $F^{\prime}$ of $F$ in $K$ with t.d. $\left(F^{\prime} / F\right)=1$ forms a geometry under algebraic dependence. We denote this geometry by $G(K / F)$, and impose the minor restriction that t.d. $(K / F) \geq 3$ to avoid trivialities.

Question. If $F^{\prime}, F^{\prime \prime}, K^{\prime}, K^{\prime \prime} \models a c f_{p}$, when is $G\left(K^{\prime} / F^{\prime}\right) \simeq G\left(K^{\prime \prime} / F^{\prime \prime}\right)$ ?

Since t.d. $(K / F)-1$ is the dimension of the geometry $G(K / F)$, it is clearly necessary that t.d. $\left(K^{\prime} / F^{\prime}\right)=$ t.d. $\left(K^{\prime \prime} / F^{\prime \prime}\right)$ if the geometries are to be isomorphic. We will show that, indeed, $F^{\prime} \simeq F^{\prime \prime}$ as well, by showing that if t.d. $\left(F^{\prime \prime} / F_{p}\right)$ $>$ t.d. $\left(F^{\prime} / F_{p}\right)$, then there are projective planes of $G\left(K^{\prime \prime} / F^{\prime \prime}\right)$ that are not isomorphic to planes of $G\left(K^{\prime} / F^{\prime}\right)$. (The notion of "projective plane of" will be clarified below.)

The proof of $F^{\prime} \simeq F^{\prime \prime}$ in the case $p \neq 0$ will be based upon a partial study of the skew field of quotients, $\mathscr{O}_{F}$, of the ring of $p$-polynomials in one variable over $F$. (The approach of distinguishing $G\left(K^{\prime} / F^{\prime}\right)$ and $G\left(K^{\prime \prime} / F^{\prime \prime}\right)$ by studying the $\mathscr{O}_{F}$ was suggested by Evans and Hrushovski in [2].) We will also

Received by the editors December 5, 1990 and, in revised form, April 17, 1991.

1991 Mathematics Subject Classification. Primary 03C60; Secondary 12L12.

Key words and phrases. Algebraically closed field, algebraic group, projective plane. 
show that if t.d. $\left(F / F_{p}\right) \neq 0$, then $\mathscr{O}_{F}$ and $\mathscr{O}_{\overline{F_{p}}}$ are not elementarily equivalent in the language of rings.

We begin by reviewing some terminology and results of Evans and Hrushovski in [2] concerning the characterization of projective planes in $G(K / F)$. The results are given in [2] for projective geometries of arbitrarily large dimension. We restrict them to planes only for the sake of brevity.

We call a 6-tuple of points $(X, Y, Z, U, V, W)$ of $G(K / F)$ a partial quadrangle if the six points are coplanar and the only collinear triples among them are $X U Y, X Z W, Z V Y$, and $U V W$. Evans and Hrushovski establish the following representation result.

Theorem 0.1. If $(X, Y, Z, U, V, W)$ is a partial quadrangle of $G(K / F)$, then there are an irreducible, one-dimensional algebraic group $(G, *)$ in some $K^{n}$, definable over $F$, and generics $\bar{x}, \bar{y}$, and $\bar{z}$ of $G$ such that $X=\operatorname{acl}_{F}(\bar{x})$, $Y=\operatorname{acl}_{F}(\bar{y}), Z=\operatorname{acl}_{F}(\bar{z}), U=\operatorname{acl}_{F}(\bar{x} * \bar{y}), V=\operatorname{acl}_{F}(\bar{z} * \bar{y})$, and $W=$ $\operatorname{acl}_{F}\left(\bar{x} * \bar{z}^{-1}\right)$.

The irreducible, one-dimensional algebraic groups definable over $F$ are well known, and are all isomorphic, as algebraic groups, to one of the following commutative groups:

(i) $(F,+)$,

(ii) $\left(F^{*}, \cdot\right)$,

(iii) an elliptic curve with the usual group operation.

Fix a partial quadrangle $(X, Y, Z, U, V, W)$, and find $G, \bar{x}, \bar{y}, \bar{z}$ as in the theorem. Since $G$ is commutative, the $\operatorname{group} \operatorname{Hom}(G, G)$ of morphisms of $G$ (as an algebraic group) may be given a ring structure $(\operatorname{Hom}(G, G),+, \circ)$, where $\circ$ is composition and $f+g$ is given by $(f+g)(x)=f(x) * g(x)$.

Each of the rings $(\operatorname{Hom}(G, G),+$, o) is known to be a left Ore domain, i.e., to be a domain satisfying

$$
\forall a, b \neq 0 \exists x, y \neq 0 a x=b y,
$$

hence, by standard results, to be embeddable in a skew field of quotients, $O(G)$.

We now clarify the notion of "projective plane of" a geometry, as used by Evans and Hrushovski. If $(G, \mathrm{cl})$ is a geometry, $H \subseteq G$ and $\mathrm{cl}^{*}$ is defined on $\mathscr{P}(H)$ by $\operatorname{cl}^{*}(X)=\operatorname{cl}(X) \bigcap H$, then $\left(H, \mathrm{cl}^{*}\right)$ is again a geometry. We will say that $\left(H, \mathrm{cl}^{*}\right)$ is a projective plane of $(G, \mathrm{cl})$, or simply that $H$ is a projective plane of $(G, \mathrm{cl})$, if, considered by itself as a geometry, $\left(H, \mathrm{cl}^{*}\right)$ is a projective plane.

Evans and Hrushovski prove the following theorem.

Theorem 0.2. Let $(G, *)$ be an irreducible, one-dimensional algebraic group definable over $F$, and let $\bar{x}, \bar{y}, \bar{z}$ be independent generics of $G$. Then the following hold:

(1) If $G$ is considered to be a $\operatorname{Hom}(G, G)$ module, then in the free submodule generated by $\bar{x}, \bar{y}, \bar{z}$, elements are dependent with respect to $\operatorname{Hom}(G, G)$ 
exactly if they are acl $l_{F}$ dependent. Thus, the set

$$
P(G ; \bar{x}, \bar{y}, \bar{z})=\left\{\operatorname{acl}_{F}(\alpha \bar{x} * \beta \bar{y} * \gamma \bar{z}): \alpha, \beta, \gamma \in \operatorname{Hom}(G, G) \text { not all zero }\right\}
$$

is a projective plane of $G(K / F)$ coordinatized by $O(G)$.

(2) If $P$ is any projective plane of $G(K / F)$ containing the points of the partial quadrangle $\left(\operatorname{acl}_{F}(\bar{x}), \operatorname{acl}_{F}(\bar{y}), \operatorname{acl}_{F}(\bar{z}), \operatorname{acl}_{F}(\bar{x} * \bar{y}), \operatorname{acl}_{F}(\bar{z} * \bar{y})\right.$, $\left.\operatorname{acl}_{F}\left(\bar{z} * \bar{x}^{-1}\right)\right)$, then $P$ is a subplane of $P(G ; \bar{x}, \bar{y}, \bar{z})$, whence $P$ is coordinatized by a sub-skew-field of $O(G)$.

We list the possibilities for $O(G)$ (for references, see [2]). $\operatorname{char}(F)=0$.

(1) If $G \simeq(F,+)$, then $O(G) \simeq F$.

(2) If $G \simeq\left(F^{*}, \cdot\right)$, then $O(G) \simeq \mathbf{Q}$.

(3) If $G$ is an elliptic curve, then $O(G) \simeq \mathbf{Q}(\sqrt{-d})$ for some $d \in \mathbf{N}$. $\operatorname{char}(F)=p \neq 0$.

(1) If $G \simeq(F,+)$, then $\operatorname{Hom}(G, G)$ is isomorphic to the ring of polynomials $\sum_{i} \alpha_{i} Y^{p^{i}} \in F[Y]$ under polynomial addition and composition. We denote this ring by $\mathscr{R}_{F}$ and its skew field of quotients $O(G)$ by $\mathscr{O}_{F}$.

(2) If $G \simeq\left(F^{*}, \cdot\right)$, then $O(G) \simeq \mathbf{Q}$.

(3) If $G$ is an elliptic curve, then $O(G) \simeq \mathbf{Q}(\sqrt{-d})$ for some $d \in \mathbf{N}$, or $O(G)$ is isomorphic to a quaternion algebra over $\mathbf{Q}$. (There are only a finite number of possibilities for $d$. See, e.g., [4] for a full analysis.)

Since any projective plane in $G(K / F)$ contains the points of a partial quadrangle of $G(K / F)$, we have the following.

Corollary 0.3. A projective plane is isomorphic to a plane of $G(K / F)$ if and only if it is coordinatizable by a sub-skew-field of one of the skew fields listed above.

Now let us return to our original question. Suppose that $F^{\prime}$ and $F^{\prime \prime}$ are two nonisomorphic algebraically closed fields of characteristic $p$. Without loss of generality, we may assume that $F^{\prime}$ is a subfield of $F^{\prime \prime}$. In order to show that $G\left(K^{\prime} / F^{\prime}\right)$ and $G\left(K^{\prime \prime} / F^{\prime \prime}\right)$ are not isomorphic, it suffices to show that some skew field in the list for $F^{\prime \prime}$ is not a sub-skew-field of any of the skew fields listed for $F^{\prime}$.

In the case $p=0$, each of the skew fields for $F^{\prime}$ is, in fact, a subfield of $F^{\prime}$, so we simply note that $F^{\prime \prime}$ is not a subfield of $F^{\prime}$.

The case $p \neq 0$ is less straightforward. Let $G^{\prime \prime}$ be the additive group of $F^{\prime \prime}$. Then $O\left(G^{\prime \prime}\right) \simeq \mathscr{O}_{F^{\prime \prime}}$. Since $F^{\prime \prime}$ is (isomorphic to) a sub-skew-field of $\mathscr{O}_{F^{\prime \prime}}$, there is a projective plane $P$ of $G\left(K^{\prime \prime} / F^{\prime \prime}\right)$ coordinatized by $F^{\prime \prime}$. In order to show that $P$ is not isomorphic to a plane of $G\left(K^{\prime} / F^{\prime}\right)$, it is therefore sufficient to show that $F^{\prime \prime}$ is not a subfield of any of the skew fields listed for $F^{\prime}$. Since $\mathscr{O}_{F^{\prime}}$ is the only one of these of characteristic $p$, the problem reduces to showing that $F^{\prime \prime}$ is not contained in $\mathscr{O}_{F^{\prime}}$. The proof of this fact and the analysis of the skew fields $\mathscr{O}_{F}$ necessary to arrive at the proof constitute the main results of this paper. 


\section{Preliminaries}

Although many of the definitions and preliminary results hold for arbitrary fields of nonzero characteristic, specifying which do not lead to distinctions of any interest in the present context. Therefore, for the remainder of the paper, $F$ will be an algebraically closed field of nonzero characteristic $p$.

Definition 1.1. $\mathscr{R}_{F}=\left(R_{F},+, *\right)$, where

- $R_{F}=\left\{\alpha_{0} X+\alpha_{1} X^{p}+\cdots+\alpha_{k} X^{p^{k}}: k \geq 0, \alpha_{i} \in F\right.$ for all $\left.i\right\}$;

- + is polynomial addition;

- * is composition.

$\mathscr{R}_{F}$ is called the ring of p-polynomials over $F$.

We now provide another representation of $\mathscr{R}_{F}$ that is easier to work with in the context of this paper.

Definition 1.2. $\mathscr{S}_{F}=\left(S_{F},+, \circ\right)$, where

- $S_{F}$ is the set of formal polynomials in $X$ over $F$;

- $+: S_{F} \times S_{F} \rightarrow S_{F}$ is polynomial addition;

- $\circ: S_{F} \times S_{F} \rightarrow S_{F}$ is defined by

$$
\begin{gathered}
\left(\alpha_{0}+\alpha_{1} X+\cdots+\alpha_{k} X^{k}\right) \circ\left(\beta_{0}+\beta_{1} X+\cdots+\beta_{m} X^{m}\right) \\
=\gamma_{0}+\gamma_{1} X+\cdots+\gamma_{k+m} X^{k+m},
\end{gathered}
$$

where $\gamma_{l}=\sum_{i+j=l} \alpha_{i} \beta_{j}^{p^{i}}$.

Theorem 1.1. $\mathscr{R}_{F} \simeq \mathscr{S}_{F}$.

Proof. We leave it to the reader to verify that the map $\sigma: R_{F} \rightarrow S_{F}$ defined by $\alpha_{0} X+\cdots+\alpha_{k} X^{p^{k}} \mapsto \alpha_{0}+\alpha_{1} X+\cdots+\alpha_{k} X^{k}$ is a ring isomorphism.

Henceforth, we shall work with $\mathscr{S}_{F}$. Routine calculations show the following.

Proposition 1.2. (1) $\mathscr{S}_{F}$ is a ring with unity $1_{F}$, zero $0_{F}$, and no zero divisors.

(2) $(F,+, \circ \mid F \times F)=(F,+, \cdot)$, where $\cdot$ is the usual multiplication on $F$.

(3) $\circ\left|F \times S_{F}=\cdot\right| F \times S_{F}$, where $\cdot$ is the usual multiplication on $F[X]$.

(4) $\circ\left|S_{F} \times\{X\}=\cdot\right| S_{F} \times\{X\}$.

(5) $\mathscr{S}_{F}$ is generated as a ring by $\{X\} \cup F$ and

$$
\alpha_{0}+\alpha_{1} X+\cdots+\alpha_{k} X^{k}=\alpha_{0}+\alpha_{1} \operatorname{circ} X+\cdots+\alpha_{k} \circ \underbrace{X \circ \cdots \circ X}_{k} .
$$

Remark. $\mathscr{S}_{F}$ is the unique ring on $S_{F}$ satisfying

- addition is polynomial addition;

- (3) and (4) of Proposition 1.2 hold;

- $X^{m} \circ \alpha=\alpha^{p^{m}} X^{m}$ for all $m \geq 0$ and all $\alpha$ in $F$.

This characterization of $\mathscr{S}_{F}$ is probably the most intuitive.

Throughout, if $R$ is any ring, then $R^{*}$ denotes $R \backslash\{0\}$. 
Definition 1.3. A ring $R$ with no zero divisors is a left Ore domain if for any $x, y \in R^{*}$, there are $z, w \in R^{*}$ with $z x=w y$.

By a theorem of Ore, any left Ore domain $D$ embeds in a (unique) left skew field of quotients, i.e., a skew field $\mathscr{O}(D) \geq D$ such that each element $x$ of $\mathscr{O}(D)$ satisfies $x=b^{-1} a$ for some $a, b \in D$. Thus, if we write $a / b=b^{-1} a$ for $a, b \in D$, then $O(D)=\{a / b: a, b \in D$, and $b \neq 0\}$.

It follows from standard ring theory results that $\mathscr{S}_{F}$ is a left Ore domain. Alternatively, an elementary proof may be found in [3]. So we may define

Definition 1.4. $\mathscr{O}_{F}=O\left(\mathscr{S}_{F}\right)$.

Let deg: $S_{F} \rightarrow \mathbf{N} \cup\{-\infty\}$ be the polynomial degree function, where $\operatorname{deg}(0)$ $=-\infty$. It is easily seen that deg behaves nicely, i.e.,

Lemma 1.3. Let $a, b \in S_{F}$. Then

(1) $\operatorname{deg}(a \circ b)=\operatorname{deg}(a)+\operatorname{deg}(b)$;

(2) $\operatorname{deg}(a+b) \leq \max (\operatorname{deg}(a), \operatorname{deg}(b))$, and < holds if and only if $\operatorname{deg}(a)=$ $\operatorname{deg}(b)$ and the leading coefficients of $a$ and $b$ are negatives of one another.

We may extend the degree function on $\mathscr{S}_{F}$ to a degree function on $\mathscr{O}_{F}$ by setting $\operatorname{deg}(a / b)=\operatorname{deg}(a)-\operatorname{deg}(b)$.

The proof that deg is well defined on $\mathscr{O}_{F}$ is elementary, and we leave it to the reader, along with the proof of the following extension of Lemma 1.3 to $\mathscr{O}_{F}$.

Lemma 1.4. Let $z, w \in O_{F}$. Then

(1) $\operatorname{deg}(z \circ w)=\operatorname{deg}(z)+\operatorname{deg}(w)$;

(2) $\operatorname{deg}(z+w) \leq \max (\operatorname{deg}(z), \operatorname{deg}(w))$, and strict inequality holds only if $\operatorname{deg}(z)=\operatorname{deg}(w)$.

Definition 1.5. If $B \subseteq O_{F}$ and $R$ is a subring of $O_{F}$, then $Z_{R}(B)$ is the centralizer of $B$ in $R$. When $R$ is $O_{F}$ we write simply $Z(B)$.

The proof of the following lemma is standard, but we include it to familiarize the reader with the details particular to $\mathscr{S}_{F}$.

Lemma 1.5. For any $a, b \in S_{F}, b \neq 0$, there are $r, c \in S_{F}$ with $\operatorname{deg}(r)<$ $\operatorname{deg}(b)$ and $a=r+b \circ c$.

Proof. If $\operatorname{deg}(a)<\operatorname{deg}(b)$, set $a=r, c=0$.

Now suppose the proposition holds for all $a^{\prime}, b^{\prime}$ with $\operatorname{deg}\left(a^{\prime}\right)-\operatorname{deg}\left(b^{\prime}\right)<$ $m$, and set $a=\alpha_{0}+\alpha_{1} X+\cdots+\alpha_{k+m} X^{k+m}$, where $\alpha_{k+m} \neq 0$ and $b=$ $\beta_{0}+\beta_{1} X+\cdots+\beta_{k} X^{k}$, where $\beta_{k} \neq 0$.

Let $\gamma$ be the $p^{k}$ th root of $\alpha_{k+m} / \beta_{k}$. Then $\beta_{k} X^{k} \circ \gamma X^{m}=\alpha_{k+m} X^{m+k}$, whence $\operatorname{deg}\left(a-b \circ \gamma X^{m}\right)<\operatorname{deg}(a)$. If $\operatorname{deg}\left(a-b \circ \gamma X^{m}\right)<\operatorname{deg}(b)$, set $r=$ $a-b \circ \gamma X^{m}, c=\gamma X^{m}$. Otherwise, by the inductive hypothesis, there are $r$ and $c^{\prime}$ in $S_{F}$ with $\operatorname{deg}(r)<\operatorname{deg}(b)$ such that $a-b \circ \gamma X^{m}=r+b \circ c^{\prime}$. Then

$$
a=r+b \circ c^{\prime}+b \circ \gamma X^{m}=r+b \circ\left(c^{\prime}+\gamma X^{m}\right) \text {. }
$$

Set $c=c^{\prime}+\gamma X^{m}$. 
Dividing through on the left by $b$, we immediately have

Lemma 1.6. For any $z \in O_{F}$, there are $s \in S_{F}$ and $z^{\prime} \in O_{F}$ with $\operatorname{deg}\left(z^{\prime}\right)<0$ and $z=z^{\prime}+s$.

Of particular importance in the work to follow are the elements of $O_{F}$ of degree zero. We note that by Lemma 1.6, such elements may be written as $z^{\prime}+\delta$, where $\operatorname{deg}\left(z^{\prime}\right)<0$ and $\delta \in F^{*}$

The proof of the following proposition follows by an easy induction from Lemma 1.4.

Proposition 1.7. For $0<i<k$, let $z_{i}=z_{i}^{\prime}+\delta_{i}$, where $\delta_{i} \in F^{*}$ and $\operatorname{deg}\left(z_{i}^{\prime}\right)<$ 0. If $q\left(Z_{1}, \ldots, Z_{k}\right) \in F_{p}\left[Z_{1}, \ldots, Z_{k}\right]$, then

$$
\operatorname{deg}\left(q\left(z_{1}, \ldots, z_{k}\right)-q\left(\delta_{1}, \ldots, \delta_{k}\right)\right)<0 .
$$

Thus, setting $z^{\prime}=q\left(z_{1}, \ldots, z_{k}\right)-q\left(\delta_{1}, \ldots, \delta_{k}\right)$ we have $q\left(z_{1}, \ldots, z_{k}\right)=$ $z^{\prime}+q\left(\delta_{1}, \ldots, \delta_{k}\right)$, with $\operatorname{deg}\left(z^{\prime}\right)<0$.

Proposition 1.8. Suppose that $z=x+c$, where $\operatorname{deg}(x)<0$ and $c \in S_{F}$. Then for all $\alpha \in F$,

$$
z \in Z(\alpha) \Leftrightarrow x \in Z(\alpha) \text { and } c \in Z(\alpha) .
$$

Proof. $(\Leftarrow)$ Trivial.

$\Rightarrow$ Suppose that $\alpha \in F$ and $z \circ \alpha=\alpha \circ z$. Then $x \circ \alpha-\alpha \circ x=\alpha \circ c-$ $c \circ \alpha$. Now $\alpha \circ c-c \circ \alpha$ is in $S_{F}$, so is either zero or has nonnegative degree. But also

$$
\operatorname{deg}(x \circ \alpha-\alpha \circ x) \leq \operatorname{deg}(x)+\operatorname{deg}(\alpha) \leq \operatorname{deg}(x)<0 .
$$

Hence, $x \circ \alpha-\alpha \circ x=\alpha \circ c-c \circ \alpha=0$. Thus, $x, c \in Z(\alpha)$.

Lemma 1.9. If $B \subseteq F$ and $z \in O_{F}$, then $z \in Z(B)$ if and only if $z=x / y$ for some $x, y \in S_{F} \cap Z(B)$.

Proof. $(\Leftarrow)$ Trivial.

$(\Rightarrow)$ Write $z=a / b, a, b \in S_{F}$. We proceed by induction on $\operatorname{deg}(a)+$ $\operatorname{deg}(b)$.

If $\operatorname{deg}(a)+\operatorname{deg}(b)=0$, then $a / b \in F$, and the result is clear.

Now suppose that the result holds for all $a / b \in Z(B)$ with $\operatorname{deg}(a)+$ $\operatorname{deg}(b)<n$, and fix $a / b \in Z(B)^{*}$ with $\operatorname{deg}(a)+\operatorname{deg}(b)=n$. Since $a / b \in Z(B)$ if and only if $b / a \in Z(B)$, we may suppose that $\operatorname{deg}(a) \geq \operatorname{deg}(b)$. Then there are $r$ and $c$ in $S_{F}$ with $\operatorname{deg}(r)<\operatorname{deg}(b)$ and $a / b=r / b+c$. By Proposition 1.8, $r / b$ and $c$ are in $Z(B)$. But $\operatorname{deg}(r)+\operatorname{deg}(b)<\operatorname{deg}(a)+\operatorname{deg}(b)$, so by the inductive hypothesis, $r / b=x^{\prime} / y$ for some $x^{\prime}$ and $y$ in $Z(B) \cap S_{F}$. Then

$$
a / b=r / b+c=x^{\prime} / y+c=\left(x^{\prime}+y \circ c\right) / y .
$$

Set $x=x^{\prime}+y \circ c$. Then since $x^{\prime}, y, c \in Z(B) \cap S_{F}$, we have $x, y \in Z(B) \cap$ $S_{F}$ and $a / b=x / y$.

Corollary 1.10. If $t \in F$ is transcendental, then $Z(t)=F$.

Proof. Clearly, $Z_{S_{F}}(t)=F$. The result follows immediately from Lemma 1.9. 


\section{MAIN Results}

Fix $F$. If $\alpha \in F$, then $\alpha$ has a $2^{n}$ th root in $\mathscr{O}_{F}$ for arbitrary $n$. By a simple induction based on 1 of Lemma 1.4, we have, as a partial converse, that if $z \in \mathscr{O}_{F}$ has arbitrary $2^{n}$ th roots in $\mathscr{O}_{F}$, then $z=0$ or $\operatorname{deg}(z)=0$.

We may now easily prove our first main result.

Theorem 2.1. If t.d. $\left(F^{\prime \prime} / F_{p}\right)>$ t.d. $\left(F^{\prime} / F_{p}\right)$, then $F^{\prime \prime}$ does not embed in $\mathscr{O}_{F^{\prime}}$.

Proof. Since $\left|\mathscr{O}_{F}\right|=|F|$ for any $F$, the result is nontrivial only for $F^{\prime}$ and $F^{\prime \prime}$ countable. Moreover, since an embedding of an algebraically closed field of infinite transcendence degree over $F_{p}$ would immediately give embeddings of all fields of finite transcendence degree over $F_{p}$, it suffices to take $F^{\prime \prime}$, hence $F^{\prime}$, of finite transcendence degree over $F_{p}$. So suppose that t.d. $\left(F^{\prime} / F_{p}\right)=m<$ $n=\mathrm{t} . \mathrm{d} .\left(F^{\prime \prime} / F_{p}\right)$, and assume, for a contradiction, that we have an embedding $\tau$ of $F^{\prime \prime}$ into $\mathscr{\sigma}_{F^{\prime}}$.

Let $t_{1}, \ldots, t_{n}$ be independent transcendentals from $F^{\prime \prime}$. Since each $t_{i}$ has arbitrary $2^{n}$ th roots in $F^{\prime \prime}$, so does each $\tau\left(t_{i}\right)$ in $\tau\left(F^{\prime \prime}\right)$, hence in $\mathscr{O}_{F^{\prime}}$. Since $\tau$ is a ring homomorphism, each $\tau\left(t_{i}\right)$ is nonzero. Hence, as noted in the remarks above, each $\tau\left(t_{i}\right)$ has degree zero, and we may write $\tau\left(t_{i}\right)=z_{i}+\delta_{i}$ for some $\delta_{i} \in F^{\prime}$ and $z_{i} \in \mathscr{O}_{F^{\prime}}$ with $\operatorname{deg}\left(z_{i}\right)<0$.

Since t.d. $\left(F^{\prime}\right)=m<n, \delta_{1}, \ldots, \delta_{n}$ are algebraically dependent over $F_{p}$. Hence, there is a nonzero polynomial $q\left(Z_{1}, \ldots, Z_{n}\right) \in F_{p}\left[Z_{1}, \ldots, Z_{n}\right]$ such that $q\left(\delta_{1}, \ldots, \delta_{n}\right)=0$.

Since $t_{1}, \ldots, t_{n}$ are independent transcendentals, $q\left(t_{1}, \ldots, t_{n}\right)$ is also a transcendental. Thus, repeating the argument used for $t_{i}, \operatorname{deg}\left(\tau\left(q\left(t_{1}, \ldots, t_{n}\right)\right)\right)$ $=0$. On the other hand, by Proposition 1.7, for some $z^{\prime} \in \mathscr{O}_{F^{\prime}}$ of degree less than zero, we have

$$
q\left(\tau\left(t_{1}\right), \ldots, \tau\left(t_{n}\right)\right)=q\left(\delta_{1}, \ldots, \delta_{n}\right)+z^{\prime}=0+z^{\prime}=z^{\prime},
$$

so $\tau\left(q\left(t_{1}, \ldots, t_{n}\right)\right)=q\left(\tau\left(t_{1}, \ldots, t_{n}\right)\right)=z^{\prime}$ has negative degree, a contradiction.

As noted in the introduction, we have proved

Corollary 2.2. If $F, F^{\prime}, K$, and $K^{\prime}$ are algebraically closed fields of the same characteristic and $G(K / F) \simeq G\left(K^{\prime} / F^{\prime}\right)$, then $K: F \simeq K^{\prime}: F^{\prime}$.

We now turn to the question of elementary equivalence of the $\mathscr{O}_{F}$. Fix $F$ of positive transcendence degree over $F_{p}$. Theorem 2.1 gives us immediately that $\mathscr{O}_{F}$ does not embed in $\mathscr{O}_{\overline{F_{p}}}$, since $F$ does not. The proof of Theorem 2.1 in this case may be summarized by saying that $F$, hence $\mathscr{O}_{F}$, has an element $t$ satisfying 'any nontrivial polynomial in $t$ has degree 0 ', while $\mathscr{O}_{\overline{F_{p}}}$ does not. There is no obvious way of writing this property as a first order formula. We can, however, define a (possibly) smaller set that still contains any transcendental. Let $\varphi(x)$ be the formula

$$
\forall z\left(z x=x z \rightarrow \exists w\left(w x=x w \& w^{2}=z\right)\right) .
$$


By Corollary 1.7 we easily have

Proposition 2.3. If $t \in F$ is transcendental over $F_{p}$, then $\mathscr{O}_{F} \vDash \varphi(t)$. Thus, if t.d. $\left(F / F_{p}\right)>0$ then $\mathscr{O}_{F} \vDash \exists x \varphi(x)$.

On the other hand, $\mathscr{O}_{\overline{F_{p}}}$ has no element satisfying $\varphi$.

Proposition 2.4. $\mathscr{O}_{\overline{F_{p}}} \models \neg \exists x \varphi(x)$.

Proof. If $\alpha \in \overline{F_{p}}$, then there is $k>0$ such that $\alpha^{p^{k}}=\alpha$, whence $X_{k} \alpha=$ $\alpha^{p^{k}} X^{k}=\alpha X$. Thus, every element of $\overline{F_{p}}$ is centralized by some positive power of $X$. It follows easily that every element of $\mathscr{\sigma}_{\overline{F_{p}}}$ is centralized by some positive power of $X$. Thus, the centralizer of any element of $\mathscr{O}_{\overline{F_{p}}}$ has a nonzero element of nonzero degree. But for any $y \in \mathscr{O}_{\overline{F_{p}}}$, if $\varphi(y)$ holds, then every element of $Z(y)$ has arbitrary $2^{n}$ th roots, whence every element of $Z(y)$ is either zero or has degree zero. Thus, no element of $\mathscr{O}_{\overline{F_{p}}}$ can satisfy $\varphi$.

We now have the desired result.

Corollary 2.5. If $F$ has positive transcendence degree over $F_{p}$, then $\mathscr{O}_{F}$ and $\mathscr{O}_{\overline{F_{p}}}$ are not elementarily equivalent.

\section{CONCLUDING REMARKS}

1. An independent proof of Corollary 2.2 and more extensive results about modular sublattices of (the lattice associated with) $G(K / F)$ (a weaker notion than that of modular subgeometry) can be obtained in the characteristic zero case by making use of the Lie algebra of derivations of $K$ which annihilate $F$, whose lattice of sub-Lie algebras dually embeds to $G(K / F)$ (see [1] for the first work along this line). I develop these in my thesis.

2. I do not know whether Corollary 2.5 can be extended to arbitrary pairs of models of $a c f_{p}$ of distinct, finite transcendence degrees over the prime field.

\section{REFERENCES}

1. C. J. Ash and John W. Rosenthal, Intersections of algebraically closed fields, Ann. Pure Appl. Logic 30 (1986), 103-119.

2. D. Evans and E. Hrushovski, Projective planes in algebraically closed fields, Proc. London Math. Soc. (3) 62 (1991), 1-24.

3. B. Lindström, On p-polynomial representations of projective geometries in algebraic combinatorial geometries, Math. Scand. 63 (1988), 36-42.

4. J. Silverman, The arithmetic of elliptic curves, Springer-Verlag, Berlin and New York, 1986.

Department of Mathematical Sciences, University of Illinois at Chicago, P.O. Box 4348, Chicago, ILlinois 60680 